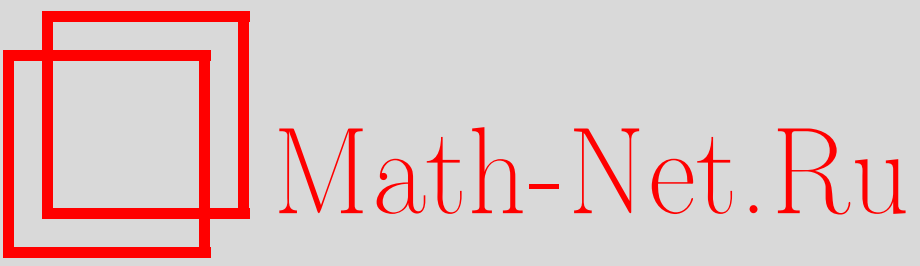

М. Д. Абловиц, К. Аренс, Д. Бьондини, С. Чакраварти, Э. Докерти, Компенсация дисперсии за счет периодической групповой задержки уменьшает индуцированные столкновениями временные сдвиги в квазилинейных системах с управлением дисперсией, ТМФ, 2005, том 144, номер 1, 6-13

DOI: https://doi.org/10.4213/tmf1826

Использование Общероссийского математического портала Math-Net.Ru подразумевает, что вы прочитали и согласны с пользовательским соглашением

http://www . mathnet.ru/rus/agreement

Параметры загрузки:

IP : 3.80 .181 .102

26 апреля 2023 г., 10:00:50 
ТЕОРЕТИЧЕСКАЯ

И МАТЕМАТИЧЕСКАЯ

ФИЗИКА

Том 144, № 1

июль, 2005

(C) 2005 г.

М. Дж. Абловиц*, К. Аренс*,

Дж. Бьондини*, С. Чакраварти*, Э. Докерти*

\section{КОМПЕНСАЦИЯ ДИСПЕРСИИ ЗА СЧЕТ ПЕРИОДИЧЕСКОЙ ГРУППОВОЙ ЗАДЕРЖКИ УМЕНЬШАЕТ ИНДУЦИРОВАННЫЕ СТОЛКНОВЕНИЯМИ ВРЕМЕННЬ́ІЕ СДВИГИ В КВАЗИЛИНЕЙНЫХ СИСТЕМАХ С УПРАВЛЕНИЕМ ДИСПЕРСИЕЙ}

Компенсирующие дисперсию модули с периодической групповой задержкой (ПГЗ) были недавно предложены в качестве механизмов для уменьшения индуцированных столкновениями временны́х сдвигов в системах с управлением дисперсией. Получены частотный и временной сдвиги в квазилинейных системах с управлением дисперсией с ПГЗ и показано, что значительное уменьшение достигается, когда даже малая доля полной дисперсии компенсируется за счет ПГЗ.

Ключевые слова: нелинейное уравнение Шредингера, оптико-волоконные коммуникации, нелинейная оптика, периодическая групповая задержка, временной сдвиг, управление дисперсией, квазилинейные системы.

\section{1. ВВЕДЕНИЕ}

Индуцированные столкновениями временны́е сдвиги, т.е. изменения времени прихода сигнала вследствие взаимодействия с сигналами в соседних частотных каналах, являются одной из главных проблем в осуществляющих включение-выключение (on-offkeying) системах с многоканальным разделением по длине волны (wavelength-divisionmultiplexed systems, WDM). Проблема оказьвается сложной, если используется управление дисперсией: из-за периодического изменения знака хроматической дисперсии в зависимости от расстояния импульсы в различных частотных каналах претерпевают зигзагообразное движение по отношению друг к другу, что приводит к взаимодействиям, состоящим из последовательности "мини-столкновений." Более того, малое значение средней дисперсии в этих системах приводит к большим длинам столкновений, озна-

* Department of Applied Mathematics, University of Colorado at Boulder, Boulder, CO 803090526, USA.E-mail: markjab@boulder.colorado.edu, ahrensc@colorado.edu, biondini@buffalo.edu, chuck@crestone.uccs.edu, docherty@colorado.edu 
чаюшим, что взаимодействие разворачивается на больших расстояниях. Действительно, в то время как хорошо известно, что умеренное управление дисперсией может давать выигрьш в отношении взаимодействий между каналами, в предельных случаях процесс столкновений может привести к взаимному разрушению двух WDM-импульсов [1].

Недавно было предложено использовать модули с периодической групповой задержкой (ПГЗ) как средство уменьшения индуцированных столкновениями временны́х сдвигов [2]-[4]. ПГЗ-модули [5]-[7] служат для компенсации некоторой доли дисперсии, накапливаемой на каждом участке дисперсионного отображения, причем компенсация остаюшейся доли дисперсии по-прежнему достигается за счет компенсируюших дисперсию волокон. Преимушество такого подхода к солитонным системам с управлением дисперсией было продемонстрировано как в численных экспериментах [2], [4], так и в прямых экспериментах по передаче сигнала [3]. Цель данной статьи двояка. Во-первых, мы выведем выражения для значения индуцированных столкновениями частотных и временны́х сдвигов в квазилинейных системах с возвратом к нулю, использующих ПГЗ. Во-вторых, мы используем как наши аналитические результаты, так и прямое численное моделирование, для того чтобы показать, что использование ПГЗ высокоэффективно как средство уменьшения индуцированных столкновениями временны́х сдвигов в квазилинейных системах с возвратом к нулю. Как и в случае солитонов, основной механизм уменьшения временно́го сдвига состоит в радикальном уменьшении длины столкновения [2]. Мы также покажем, что сами по себе ПГЗ дают и значительный прямой эффект, которьй обеспечивает восстанавливающую силу для временно́го сдвига.

\section{2. РАСПРОСТРАНЕНИЕ ИМПУЛЬСОВ В СИСТЕМАХ С ПГЗ}

Начнем с нелинейного уравнения Шредингера (НУШ), записанного в безразмерных переменных $t=t_{\mathrm{ret}} / t_{*}, \quad z=z_{\mathrm{lab}} / z_{*}, u=\mathcal{E} / \sqrt{g(z) P_{*}}$ и $D=-k^{\prime \prime} / k_{*}^{\prime \prime}$, где нормировочные параметры обозначены звездочкой. Здесь $\mathcal{E}$ - медленно меняюшаяся огибаюшая комплексного оптического поля, $t_{\mathrm{ret}}$ и $z_{\mathrm{lab}}$ - запаздываюшее время и длина распространения. Типичные нормировочные значения суть $P_{*}=1 \mathrm{MB}, z_{*}=z_{\mathrm{NL}}=1 /\left(\gamma P_{*}\right)=$ $400 \mathrm{\kappa м}, t_{*}=12$ пс и $k_{*}^{\prime \prime}=t_{*}^{2} / z_{*}=0.36$ пс $^{2} /$ км, где $\gamma=2.5(\text { Вт·км })^{-1}-$ нелинейный коэффициент волокна. Рассмотрим дисперсионное отображение для волокна, состояшего из двух участков (с коэффишиентами дисперсии и длинами $D_{1}>0, z_{1}$ и $D_{2}<0, z_{2}$ ) и усилителя в волокне с добавками эрбия (УВДЭ), расположенного позади второго волокна. Полная длина отображения есть $z_{a}=z_{1}+z_{2}$, а доля отображения, приходяшаяся на аномальное волокно, равна $\theta=z_{1} / z_{a}$. Здесь $g(z)$ описывает периодическое изменение мошности из-за потерь и усиления, а $D(z)$ - дисперсия локальной групповой скорости, причем обе функции являются периодическими с периодом $z_{a}$. При наличии УВДЭ имеем $g(z)=g_{0} \exp (-2 \Gamma z)$ при $n z_{a}<z<(n+1) z_{a}$, где $n$ - номер отображения, $\Gamma$ - безразмерньй коэффициент потерь, а $g_{0}=2 \Gamma z_{a} /\left(1-\exp \left(-2 \Gamma z_{a}\right)\right)$. Мера эффектов управления дисперсией выражается напряженностью отображения $[8]$ $s=\left[\left(D_{1}-\langle D\rangle\right) z_{1}-\left(D_{2}-\langle D\rangle\right) z_{2}\right] / 4$, где $\langle D\rangle=\left(D_{1} z_{1}+D_{2} z_{2}\right) / z_{a}-$ средняя дисперсия. Для одиночного импульса с нормированной частотой $\Omega_{0}$ его среднее положение $\langle t\rangle$ на 
расстоянии $z$ вдоль волокна имеет вид

$$
\langle t\rangle=\Omega_{0} \int_{0}^{z} D(x) d x=\Omega_{0}[\langle D\rangle z+C(z)],
$$

где

$$
C(z)=\int_{0}^{z}[D(x)-\langle D\rangle] d x
$$

- периодическая функция. Предположим, что два импульса $u_{ \pm}$с частотами $\pm \Omega_{0}$ изначально расположены в $\mp t_{0}$; начальное временно́е смешение $t_{0}$ связано со средним положением столкновений $z_{0}$ посредством соотношения $t_{0}=\Omega_{0}\langle D\rangle z_{0}$. Мини-столкновения между импульсами при этом возникают, когда $\left(z-z_{0}\right)\langle D\rangle+C(z)=0$. Длину столкновения можно оценить как $L_{c}=2 s /\langle D\rangle$. Полные столкновения - это те, для которых $L_{c} / 2 \leqslant z_{0} \leqslant L-L_{c} / 2$.

В системах с ПГЗ в каждый участок дисперсионного отображения вставляется ПГЗ-

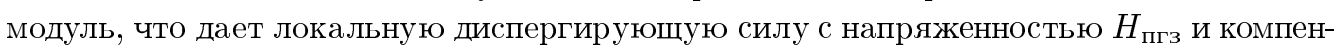
сирует некоторую долю $f$ дисперсии, обусловленной вторым волокном. Другими словами, $f=H_{\text {пгз }} /\left(D_{2} z_{2}^{\prime}+H_{\text {пгз }}\right.$ ) (здесь и далее штрих обозначает величины в системе при наличии ПГЗ). Мы будем считать, что при выполнении этой замены средняя дисперсия, отдельные дисперсии и длина отдела с первым волокном не меняются: $D_{1,2}^{\prime}=$ $D_{1,2}, z_{1}^{\prime}=z_{1}$ и $\langle D\rangle^{\prime}=\left(D_{1} z_{l}^{\prime}+D_{2} z_{2}^{\prime}+H_{\text {пгз }}\right) / z_{a}^{\prime}=\langle D\rangle$. Тогда можно найти новые параметры системы: $\theta^{\prime}=\left[\left(D_{1}-D_{2}\right)(1-f) \theta-D_{2} f\right] /\left[D_{1}(1-f)-D_{2}\right], \quad z_{a}^{\prime}=\left(\theta / \theta^{\prime}\right) z_{a}$ и $z_{2}^{\prime}=\left(1-\theta^{\prime}\right) z_{a}^{\prime}$. Полезно определить эффективную среднюю дисперсию формулой $\langle D\rangle_{\text {эфф }} \equiv\left(D_{1} z_{1}+D_{2} z_{2}^{\prime}\right) / z_{a}^{\prime}$. Используя эквивалентное определение напряженности отображения $2 s=\left(D_{1}-\langle D\rangle\right) z_{1}$, можно далее найти новую напряженность отображе-

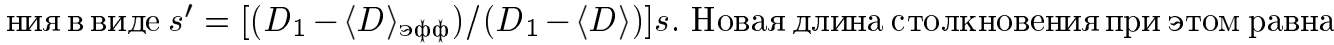

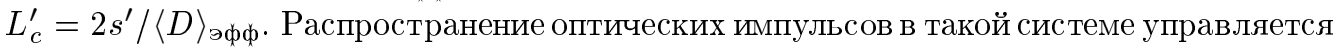
возмушенным НУШ:

$$
\begin{gathered}
i u_{z}+\frac{1}{2} D(z) u_{t t}+g(z)|u|^{2} u=i P[u], \\
\widehat{P}[u]=\left(e^{i H(\omega)}-1\right) \sum_{m=1}^{N_{a}} \delta\left(z-m z_{a}^{\prime}\right) \hat{u}(\omega, z)
\end{gathered}
$$

(cp. с [9]), где $P[u]$ выражает действие ПГЗ во временно́й области, $\widehat{P}[u]$ - преобразование Фурье от $P[u]$, а $\hat{u}(\omega, z)$ - преобразование Фурье от $u(t, z)$ и где $N_{a}$ - полное число дисперсионных отображений в передающей линии. Функция $H(\omega)$ представляет собой ПГЗ-отклик, который локально аппроксимирует квадратичный дисперсионньй профиль, но является периодическим с периодом, равным расстоянию между каналами, в нашем случае $2 \Omega_{0}$. Наконец, $\delta(z)$ - дираковская дельта-функция, которая кодирует изменение импульса на конщах ПГЗ-модуля: $\hat{u}\left(\omega, m z_{a}^{\prime+}\right)=e^{i H(\omega)} \hat{u}\left(\omega, m z_{a}^{\prime-}\right)$.

\section{3. ИНДУЦИРОВАННЫЕ СТОЛКНОВЕНИЯМИ ЧАСТОТНЫЕ И ВРЕМЕННЬ́ІЕ СДВИГИ ПРИ НАЛИЧИИ ПГЗ}

Подставляя $u=u_{+}+u_{-}$в уравнения (1) и пренебрегая четырехволновым смешиванием, находим эволюцию $u_{ \pm}$согласно уравнению (1) с дополнительным слагаемым 
$2 g(z)\left|u_{\mp}\right|^{2} u_{ \pm}$в левой части (1a). Средние время и частота импульса определяются обычными формулами

$$
\langle t\rangle=\int \frac{t\left|u_{ \pm}\right|^{2} d t}{E}, \quad\langle\Omega\rangle=-i \int \frac{u_{ \pm}^{*}\left(\partial u_{ \pm} / \partial t\right) d t}{E},
$$

где $E=\int\left|u_{ \pm}\right|^{2} d t$ - энергия импульса. (Все интегралы берутся от $-\infty$ до $\infty$, если специально не указано иное.) Используя уравнения (1) и тейлоровское приближение второго порядка для $\widehat{P}[u]$ вблизи $\omega=\Omega_{0}$ при $H\left(\Omega_{0}\right)=H^{\prime}\left(\Omega_{0}\right)=0$, найдем эволюционные уравнения для средних частоты и времени. Для $u_{+}$имеем

$$
\begin{aligned}
\frac{\partial\langle\Omega\rangle}{\partial z} & =\frac{2 g(z)}{E} \int\left|u_{+}\right|^{2} \frac{\partial\left|u_{-}\right|^{2}}{\partial t} d t, \\
\frac{\partial\langle t\rangle}{\partial z} & =D(z)\langle\Omega(z)\rangle+H_{\text {пгз }} \sum_{m=1}^{N_{a}} \delta\left(z-m z_{a}^{\prime}\right)\left(\langle\Omega\rangle-\Omega_{0}\right),
\end{aligned}
$$

где $H_{\text {пгз }}=H^{\prime \prime}\left(\Omega_{0}\right)$. Частотный и временной сдвиги тогда равны $\Delta \Omega=\Omega(z)-\Omega_{0}$ (причем $\left.\Omega(0)=\Omega_{0}\right)$ и $\Delta t(z)=\langle t(z)\rangle-\widetilde{D}(z) \Omega_{0}+t_{0}$, где $\widetilde{D}(z) \equiv \int_{0}^{z} D(x) d x$ было бы накопленной дисперсией в новой системе без ПГЗ. Удобно также определить дисперсию с учетом ПГЗ как

$$
D_{\text {пгз }}(z)=D(z)+H_{\text {пгз }} \sum_{m=1}^{N_{a}} \delta\left(z-m z_{a}^{\prime}\right)
$$

и накапливаемую дисперсию с учетом ПГЗ - как

$$
\widetilde{D}_{\text {пгз }}(z)=\int_{0}^{z} D_{\text {пгз }}(x) d x .
$$

Эволюция квазилинейного импульса с исходно гауссовым профилем $u_{ \pm}(t, 0)=$ $(a / \sqrt{2 \pi b}) \exp \left[-\left(t \pm t_{0}\right)^{2} / 2 b \pm i \Omega_{0} t\right]$ в зависимости от расстояния тогда приближенно описывается выражением [10]

$$
u_{ \pm}(t, z)=\frac{a}{\sqrt{2 \pi\left(b+i \widetilde{D}_{\text {пгз }}(z)\right)}} \exp \left[-\frac{\left(t \pm t_{0} \mp \Omega_{0} \widetilde{D}(z)\right)^{2}}{2\left(b+i \widetilde{D}_{\text {пгз }}(z)\right)} \pm i \Omega_{0} t-\frac{i}{2} \Omega_{0}^{2} \widetilde{D}(z)\right] .
$$

Используя равенство (3) в уравнениях (2), находим

$$
\Delta \Omega(L)=A \Omega_{0} \int_{0}^{L} \frac{g(z) \widetilde{D}_{0}(z)}{\left(b^{2}+\widetilde{D}_{\text {пгз }}^{2}(z)\right)^{3 / 2}} \exp \left(-2 b \Omega_{0}^{2} \frac{\widetilde{D}_{0}^{2}(z)}{b^{2}+\widetilde{D}_{\text {пгз }}^{2}(z)}\right) d z
$$

и

$$
\Delta t(L)=\widetilde{D}_{0}(L) \Delta \Omega(L)-\Delta t_{\text {ост }}+H_{\text {пгз }} \sum_{m=1}^{N_{a}} \Delta \Omega\left(m z_{a}^{\prime}\right),
$$

где $\widetilde{D}_{0}(z)=\widetilde{D}(z)-\langle D\rangle_{\text {эфф }} z_{0}$, причем $A=4 E b^{3 / 2} / \sqrt{2 \pi}$, и где "остаточный” временной

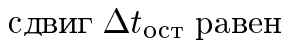

$$
\Delta t_{\text {ост }}(L)=A \Omega_{0} \int_{0}^{L} \frac{g(z) \widetilde{D}_{0}^{2}(z)}{\left(b^{2}+\widetilde{D}_{\text {пгз }}^{2}(z)\right)^{3 / 2}} \exp \left(-2 b \Omega_{0}^{2} \frac{\widetilde{D}_{0}^{2}(z)}{b^{2}+\widetilde{D}_{\text {пгз }}^{2}(z)}\right) d z .
$$


Уравнение (4б) показывает, что из-за ПГЗ имеется дополнительная восстанавливаюшая сила. Сумма в уравнении (4б) вычисляется с использованием уравнения (4a), в котором $L$ заменено на $m z_{a}^{\prime}$. Заметим, что изложенная вьше теория позволяет вычислять частотньй и временной сдвиги как для полных, так и для неполных столкновений. Заметим также, что уравнение (2б) можно записать в компактном виде как $\partial\langle t\rangle / \partial z=$ $D_{\text {пгз }}(z) \Delta \Omega(z)+D(z) \Omega_{0}$. Важно, что интегралы в уравнениях (4а) и (4в) можно эффективно брать численными квадратурами или с помошью асимптотического приближения, используя так называемый метод Лапласа (см. [11], [12]).

\section{4. АСИМПТОТИЧЕСКОЕ ПРИБЛИЖЕНИЕ ПО МЕТОДУ ЛАПЛАСА}

Интегралы в уравнениях (4а) и (4в) имеют обший вид

$$
I(\lambda)=\int_{0}^{L} F(z) e^{-\lambda \Phi(z)} d z,
$$

где $\lambda>0$ - параметр. Для больших значений $\lambda$ основной вклад в $I(\lambda)$ дает окрестность так называемой критической точки $z_{c}$, в которой $\Phi(z)$ имеет минимум, а другие области дают экспоненциально малые вклады. Функции $F(z)$ и $\Phi(z)$ можно тогда разложить в ряды Тейлора с центром в критической точке, причем, как правило, удерживаются только несколько первых членов.

В нашем случае $\Phi(z)=\widetilde{D}_{0}^{2} /\left(b^{2}+\widetilde{D}_{\text {пгз }}^{2}\right)$ и $\lambda=2 b \Omega_{0}^{2}$ как для уравнения (4а), так и для уравнения (4в). Критические точки $z_{c}$ соответствуют положениям, где происходят мини-столкновения, которые случаются при $\widetilde{D}_{0}\left(z_{c}\right)=0$. Для уравнения (4a) имеем $F(z)=$ $g \widetilde{D}_{0} /\left(b^{2}+\widetilde{D}_{\text {пгз }}^{2}\right)^{3 / 2}$, тогда как для уравнения $(4 \mathrm{~B})-F(z)=g \widetilde{D}_{0}^{2} /\left(b^{2}+\widetilde{D}_{\text {пгз }}^{2}\right)^{3 / 2}$. Таким образом, получаем $F\left(z_{c}\right)=\Phi\left(z_{c}\right)=0$ и для $\Delta \Omega$, и для $\Delta t_{\text {ост }}$, но $F^{\prime}\left(z_{c}\right) \neq 0$ для $\Delta \Omega$, тогда как $F^{\prime}\left(z_{c}\right)=0$ для $\Delta t_{\text {ост }}$. Тогда, если $z_{1}, \ldots, z_{N}$ - положения мини-столкновений, находим

$$
\begin{aligned}
\Delta \Omega(L) & \sim A \sum_{n=1}^{N}\left[\frac{F_{n}^{\prime} J_{1, n}}{b \Omega_{0} \Phi_{n}^{\prime \prime}}+\frac{3 F_{n}^{\prime \prime} \Phi_{n}^{\prime \prime} J_{2, n}-2 F_{n}^{\prime} \Phi_{n}^{\prime \prime \prime} J_{4, n}}{6 b^{3 / 2} \Omega_{0}^{2}\left(\Phi_{n}^{\prime \prime}\right)^{5 / 2}}\right], \\
\Delta t_{\text {oст }}(L) & \sim \frac{A}{b^{3 / 2} \Omega_{0}^{2}} \sum_{n=1}^{N} \frac{F_{n}^{\prime \prime} J_{2, n}}{2\left(\Phi_{n}^{\prime \prime}\right)^{3 / 2}}
\end{aligned}
$$

где $F_{n}^{\prime}=F^{\prime}\left(z_{n}\right)$ и т.д., и

$$
J_{k, n}=\int_{x_{n}^{-}}^{x_{n}^{+}} x^{k} e^{-x^{2}} d x,
$$

причем пределы интегрирования $x_{n}^{ \pm}$зависят от положений мини-столкновений. Интегралы $J_{k, n}$ можно вычислить точно в терминах хорошо известных функций. Уравнения (5) показывают, что остаточный сдвиг частоты и остаточный временной с двиг суть $\mathcal{O}\left(1 / \Omega_{0}\right)$ и $\mathcal{O}\left(1 / \Omega_{0}^{2}\right)$, соответственно. Точность метода Лапласа можно еше улучшить, если удерживать члены высших порядков. 


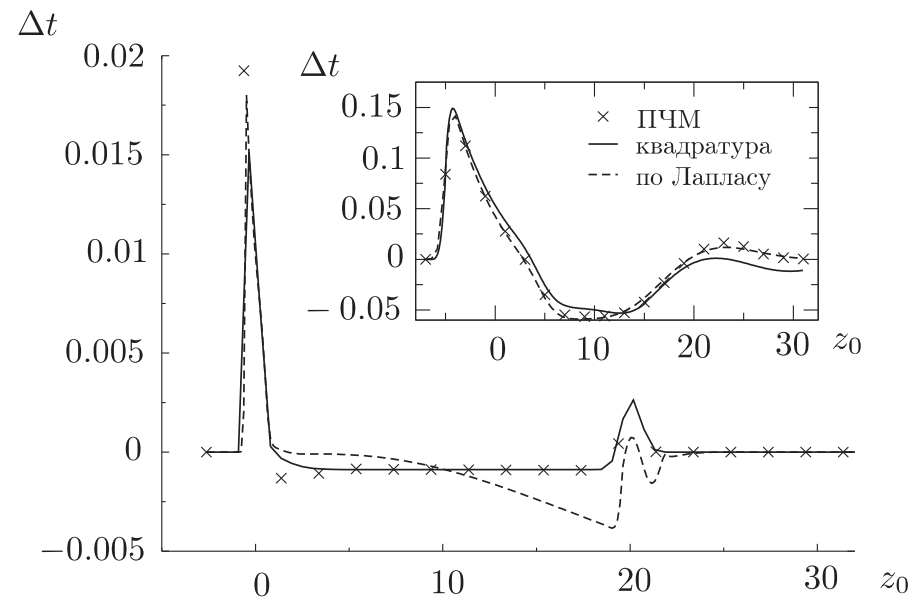

Рис. 1. Полный временной сдвиг в зависимости от среднего положения столкновений $z_{0}$ при $f=0.1$, полученный из прямого численного моделирования (ПЧМ) уравнения (1), посредством численного интегрирования (квадратура) уравнений (4) и посредством их аппроксимации (по Лапласу) с помощью уравнений (5). Врезка показывает систему без ПГЗ, $f=0$.

\section{5. ЧИСЛЕННОЕ МОДЕЛИРОВАНИЕ И ОБСУЖДДЕНИЕ}

Сравним результаты прямого численного моделирования НУШ (1), результаты численного интегрирования уравнений (4) (квадратуры) и их асимптотическую аппроксимацию с помошью уравнений (5) (метод Лапласа). В нашем квазилинейном анзаце неявно предполагается, что ПГЗ-отклик аппроксимирует квадратичньй дисперсионный профиль. Мы, таким образом, использовали кусочно-периодическую функцию, для того чтобы моделировать ПГЗ в уравнениях $(1): H(\omega, z)=\left(\omega-\Omega_{0}\right)^{2} H_{\text {пгз }} / 2$ при $2 n \Omega_{0} \leqslant \omega<2(n+1) \Omega_{0}$. Поскольку предполагается, что ПГЗ действует локально в пространстве, импульс распространялся линейно через содержащие ПГЗ-модули пространственные ячейки, с условием скачка, приведенным после уравнений (1). Таким образом, ожидается, что уравнения (4) будут хорошим приближением, когда спектральная ширина импульса мала по сравнению с периодом ПГЗ, а именно $2 \Omega_{0}$. В численных квадратурах расчет на основе уравнений (4) производился путем разбиения отрезка интегрирования $[0, L]$ на подынтервалы с постоянной дисперсией, с использованием правила трапеции на каждом подынтервале.

Мы провели численное моделирование квазилинейной системы с нормированными линейными параметрами $L=20,\langle D\rangle=0.5, s=2.5, \theta=0.5, \Gamma=9.21$ и $z_{a}=0.15$ и нормированными параметрами импульса $a=1.6, b=2$ и $\Omega_{0}=3$. Эти значения соответствуют системе с каналами, разнесенными на 80 ГГц, со средней дисперсией 0.18 пс $^{2} / \mathrm{kм}$, полной длиной передачи $8000 \mathrm{kм}$, коэффициентом потерь 0.2 дБ/км, разнесением усилителей на 40 км без ПГЗ и входным импульсом с пиковой мошностью 0.6 мВт и полной шириной на половине максимума 28.26пс.

На рис. 1 показан полный временной сдвиг $\Delta t(L)$ на выходе в зависимости от среднего положения столкновений $z_{0}$ для системы с ПГЗ $(f=0.1)$ и без ПГЗ $(f=0)$. На рис. 2 


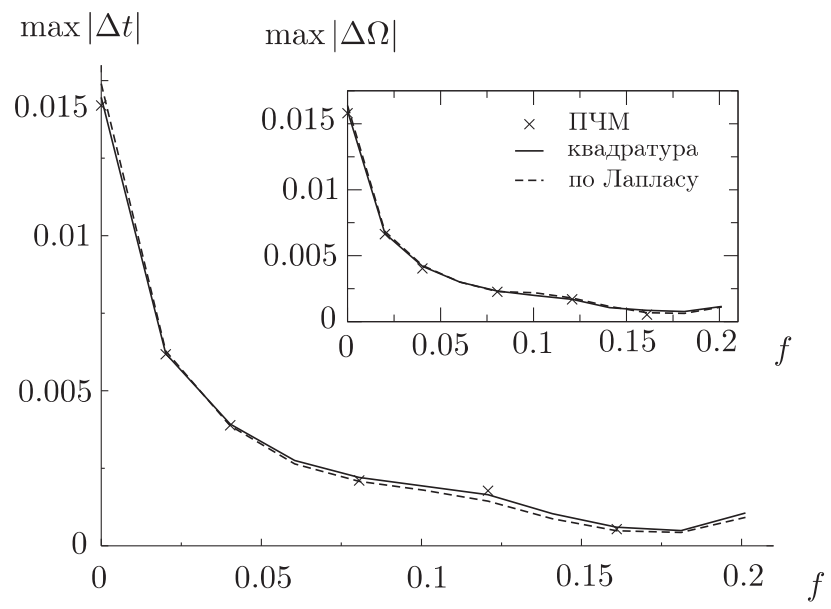

Рис. 2. Максимальные временной сдвиг и частотный сдвиг (на врезке) как функции $f$ относительно среднего положения столкновений $z_{0}$.

показано максимальное абсолютное значение полного временно́го сдвига и остаточного сдвига частоты как функций $f$ - доли компенсации, осуществляемой ПГЗ; максимум берется по отношению к среднему положению столкновений $z_{0}$.

Помимо прекрасного согласия между аналитической формулой и прямым численным моделированием, эти рисунки позволяют ясно видеть значительное уменьшение индуцированных столкновениями временны́х сдвигов, возникающих при использовании ПГЗ, даже при столь малых значениях $f$ как 0.05. Заметим, что уменьшение временно́го сдвига соответствует резкому убыванию длины столкновения: на рис. $1 L_{c}^{\prime}=1.24$ при $f=0.1$, что следует сравнить с $L_{c}=10$ при $f=0$ (без ПГЗ). Заметим также, что $L_{c}^{\prime} \ll L$, когда $f$ не слишком мало, что означает, что при наличии ПГЗ почти все столкновения полные, в отличие от случая без ПГЗ. Наконец, рис. 1 показывает, что при наличии ПГЗ эффект полных столкновений оказывается практически пренебрежимым (в отличие от случая без ПГЗ), и единственные заметные временнь́е сдвиги возникают из нескольких неполных столкновений, соответствующих столкновительным центрам $z_{0}$, расположенным вблизи начала $\left(z_{0}=0\right)$ или конца $\left(z_{0}=20\right)$ передающей линии.

ПРИЛОЖЕНИЕ

\section{Доказательство асимптотического характера соотношений (5)}

Докажем, что уравнения (5) дают асимптотику интегралов (4а) и (4в). Для уравнения (5a) докажем только, что член в главном порядке дает асимптотику. Для упрощения обозначений опустим штрихи над величинами в системе с ПГЗ. Начнем с того, что запишем уравнение (5a) в виде

$$
\begin{aligned}
\Delta \Omega(L) & =A \Omega_{0} \int_{0}^{L} F(z) e^{-\lambda \Phi(z)} d z= \\
& =A \Omega_{0} \sum_{n=0}^{N_{a}-1} \int_{n z_{a}}^{(\theta+n) z_{a}} F(z) e^{-\lambda \Phi(z)} d z+\int_{(\theta+n) z_{a}}^{(n+1) z_{a}} F(z) e^{-\lambda \Phi(z)} d z,
\end{aligned}
$$


где дисперсия постоянна на каждом подынтервале $\left(n z_{a},(\theta+n) z_{a}\right)$ и $\left((\theta+n) z_{a},(n+1) z_{a}\right)$. Без потери общности рассмотрим интеграл

$$
I(\lambda)=\int_{0}^{\theta z_{a}} F(z) e^{-\lambda \Phi(z)} d z .
$$

Можно показать, что имеется максимум одна критическая точка $c$ на каждом интервале. Можно также показать, что в окрестности точки $c, 0<c<\theta z_{a}$,

$$
\begin{gathered}
F \approx F^{\prime}(c)(z-c), \\
\Phi(z) \approx \frac{\Phi^{\prime \prime}(c)}{2}(z-c)^{2} .
\end{gathered}
$$

Тогда, используя лемму Уотсона (см. [13], гл. 3), находим

$$
I(\lambda) \sim \frac{F^{\prime}(c)}{2 \Phi^{\prime \prime}(c) \lambda},
$$

или, вспоминая, что $\lambda=2 b \Omega_{0}^{2}$,

$$
I(\lambda) \sim \frac{A F^{\prime}(c)}{b \Phi^{\prime \prime}(c) \Omega_{0}}
$$

Поскольку конечная сумма асимптотических членов является асимптотической, получаем, что в главном порядке уравнение (5a) дает асимптотику для (4a). Подобный же анализ показывает, что уравнение (5б) дает асимптотику для (4в).

Благодарности. Мы благодарим У. Л. Кат за привлечение нашего внимания к ПГЗ и за многочисленные плодотворные обсуждения. Работа была частично поддержана со стороны NSF (гранты DMS-0101340, DMS-9810751, DMS-0101476 и DMS-0104337).

\section{Список литературы}

[1] M. Ablowitz, G. Biondini, E. Olson. J. Opt. Soc. Am. B. 2001. V. 15. P. 577-583.

[2] X. Wei, X. Liu, C. Xie, L. F. Mollenauer. Opt. Lett. 2003. V. 28. P. 983-985.

[3] L. F. Mollenauer, A. Grant, X. Liu. Opt. Lett. 2003. V. 28. P. 2043-2045.

[4] X. Liu, W. Wei, L.F. Mollenauer, C.J. McKinstrie, C. Xie. Opt. Lett. 2003. V. 28. P. $1412-1414$.

[5] M. Shirasaki. IEEE Photon. Technlol. Lett. 1997. V. 9. P. 1598-1600.

[6] C. K. Madsen, G. Lenz. IEEE Photon. Technlol. Lett. 1998. V. 10. P. 994-996.

[7] X. Shu, K. Sugden, K. Byron. Opt. Lett. 2003. V. 28. P. 881-883.

[8] M. J. Ablowitz, G. Biondini. Opt. Lett. 1998. V. 23. P. 1668-1670.

[9] M.J. Ablowitz, G. Biondini, S. Chakravarty, R. Horne. Opt. Commun. 1999. V. 172. P. 211-227.

[10] M. J. Ablowitz, T. Hirooka, G. Biondini. Opt. Lett. 2001. V. 26. P. 459-461.

[11] M. J. Ablowitz, A. Docherty, T. Hirooka. Opt. Lett. 2003. V. 28. P. 1191-1193.

[12] M. J. Ablowitz, A.S. Fokas. Complex Variables: Introduction and Applications. Cambridge: Cambridge Univ. Press, 1997.

[13] Ф. Олвер. Асимптотика и специальные функции. М.: Наука, 1990. 